\title{
A chat on fat in the liver
}

\author{
Christopher Day MD¹, Paul C Adams MD², Editor-in-Chief
}

$\mathrm{D}_{\mathrm{r}}^{\mathrm{r}}$ Christopher Day is the Professor of Liver Medicine and Head of the School of Clinical Medical Sciences at the University of Newcastle upon Tyne (Newcastle, United Kingdom). He has been working in the area of fatty liver disease relating to obesity and alcohol for the past 16 years, and is perhaps best known for the 'two-hit theory' of fatty liver disease pathogenesis.

PA: You cannot pick up a journal today without hearing about fatty liver, steatohepatitis and nonalcoholic steatohepatitis (NASH). Physicians have known about the liver risks relating to obesity since the days of jejunal bypass surgery. Liver biopsies are probably declining overall, and yet it seems like we have an epidemic of this disease.

CD: I believe the apparent 'epidemic' of fatty liver disease is due to two reasons. First, we know that conditions associated with fatty liver disease, namely, obesity, diabetes and the metabolic syndrome, are on the rise due to clear lifestyle factors. However, perhaps more important than this is the increasing number of liver blood tests and abdominal ultrasounds that are being performed as part of normal medical check-ups and surveillance programs, with fatty liver disease being diagnosed incidentally. We know from large surveillance studies that approximately 30\% of the North American population will have fatty liver on ultrasound, and we also know that the vast majority of the $5 \%$ to $10 \%$ of individuals with abnormal liver function tests, unrelated to alcohol or viral hepatitis, will have nonalcoholic fatty liver disease (NAFLD).

PA: We commonly see referrals of obese patients with mild liver enzyme abnormalities. We round up the usual suspects (viral hepatitis, etc), order an ultrasound that suggests fatty infiltration of the liver, and we suggest that the patient lose weight and have their family physician tune up their lipids and blood sugar. Is this enough of an investigation? Should this be a diagnosis by exclusion?

CD: Yes, NAFLD is essentially a diagnosis of exclusion in patients with typical features. Therefore, if a physician examines a typical patient with obesity, glucose intolerance, abnormal lipids and hypertension with abnormal liver function tests, fatty liver on ultrasound and negative 'routine' liver screening tests, they can be fairly confident that the diagnosis is one of NAFLD. Further investigation, including liver biopsy, would only be required if there remains a doubt about the diagnosis. For example, I may consider a liver biopsy in patients without any of the typical features, but showing fatty liver on ultrasound, or abnormal liver function tests of uncertain etiology or where they do have the associated features, but other liver blood tests might suggest an alternative or additional cause of abnormal liver function which would require treatment in its own right. Therefore, patients with positive liver autoantibodies, or biochemical and genetic evidence of hemochromatosis, may require liver biopsy for diagnosis. Patients taking potentially hepatotoxic drugs with abnormal liver function tests in the presence of NAFLD risk factors would also fall into this category.

PA: We could not possibly arrange for all of these patients to have liver biopsies. Is there another noninvasive approach that can identify the more severe cases?

CD: At present, the only definitive way to determine the stage of fatty liver disease is liver biopsy. However, there are a number of alternative methods being developed to predict the presence of advanced disease. With respect to clinical and routine laboratory variables, we know that older age (older than 45 years), presence of diabetes, increasing body mass index, low platelet counts, low albumin levels, and a ratio of aspartate aminotransferase to alanine aminotransferase greater than one, are all predictive of advanced liver disease. Algorithms based on these variables are currently being developed and validated. In the future, it is likely that the addition of serum markers of fibrosis, for example serum hyaluronic acid or procollagen III peptide, will be included in these algorithms. There are also newer imaging techniques on the horizon, for example elastography (FibroScan, Echosens, France), which may be able to accurately determine the presence and severity of fibrosis without the need for a liver biopsy.

PA: As specialized consultants, we often do not follow up with patients in our clinics. Is it reasonable to recommend medications - metformin and fibrates - and then suggest that the family physician do the follow-up?

CD: This is perhaps the most important reason for determining liver disease stage with reasonable accuracy. My policy is to discharge patients with simple fatty liver back to their family physicians with advice on managing the normal risk factors. The diagnosis of fatty liver will either be made on liver biopsy or made on the basis of the laboratory and clinical features discussed above, effectively excluding advanced disease. However, if these clinical and laboratory features suggest advanced liver disease, I would probably perform a biopsy to 
determine disease severity and continue to follow up on those patients with advanced liver disease because these are the patients who will develop liver complications in the future, including varices and hepatocellular carcinoma. I also consider it likely that in the next year or so, evidence-based treatments for advanced fatty liver disease will be available and therefore, I would like to keep these patients 'on the books' so that they can be treated appropriately when these drugs become available.

PA: A question that never goes away is the use of statin drugs in patients with fatty liver or any type of liver disease. Has the hepatotoxicity of these medications been overstated?

CD: I agree that the hepatotoxicity of statin drugs has been markedly overstated. In my experience, the vast majority of patients with abnormal liver function tests on statins have NAFLD as the cause of abnormal liver function tests rather than the statin. It is just that in most cases, the first time these patients have liver blood tests measured is when they go on statin drugs. True statin hepatotoxicity is extremely obvious in that the patients develop markedly abnormal liver function tests reasonably quickly after starting the drug, and the liver tests rapidly return to normal on cessation. Large surveys have shown that patients with abnormal liver function tests (presumably the majority of whom will have NAFLD) are no more likely to develop statin-induced hepatotoxicity than patients with normal liver function tests. My strong advice is that all patients with NAFLD should be treated with statins if they have the normal indications.

PA: Those gazing into the future have predicted a large number of NASH cirrhotic patients, thereby increasing demand on resources for liver transplantation. Have we started to see this increase?

CD: I think there is little doubt that we will see the vast number of patients with NAFLD starting to impact on our transplant programs. There has been little firm evidence as yet that NASH cirrhosis is contributing in a major way to the transplantation workload, however, on a purely anecdotal basis on my own unit, I have no doubt that we are doing increasing numbers of transplantations on patients with NASH and they are certainly contributing markedly to our patient numbers with hepatocellular carcinoma. It is of course likely that the majority of patients with NASH cirrhosis will not be submitted for liver transplantation due to their age and comorbidity, and therefore the absence of such patients from liver transplant programs should not be taken as evidence that they are not causing a major burden on the services required for end-stage liver disease patients.

PA: How do you approach the issue of alcohol use in a patient with various forms of fatty liver disease?

CD: Interestingly, the best evidence is that sensible alcohol use (two to three drinks per day for men and one to two drinks for women) actually protects patients with obesity and the metabolic syndrome from developing advanced fatty liver disease. This is probably due to the now well-established association between sensible alcohol use and reduced risk of diabetes. Therefore, there is no rationale for recommending complete abstinence from alcohol in patients with fatty liver disease. I therefore recommend drinking within sensible limits. Of course, there is no doubt that patients drinking above these limits who have classical risk factors for NAFLD are more likely to develop advanced liver disease with obesity, perhaps the single biggest predictive factor determining why certain heavy drinkers develop advanced alcoholic liver disease and others do not.

PA: Can you predict the prevalence of fatty liver in gastroenterologists?

CD: Given that the presence of fatty liver disease in the general population is approximatetly $30 \%$ and that gastroenterologists are probably more overweight than average and certainly many of them drink too much, it seems likely that this prevalence is higher. As I always say at the end of my lectures, if you must drink excessively, stay thin and if you must eat excessively, do not drink!

\section{REFERENCES}

1. Day CP. The natural history of NAFLD: Remarkably benign in the absence of cirrhosis. Gastroenterology 2005;129:375-8.

2. Cortez-Pinto H, de Moura MC, Day CP. Non-alcoholic steatohepatitis: From cell biology to clinical practice. J Hepatol 2006;44:197-208.

3. Day CP. From fat to inflammation. Gastroenterology 2006;130:207-10.

4. Day CP. Non-alcoholic fatty liver disease: Current concepts and management strategies. Clin Med 2006;6:19-25.

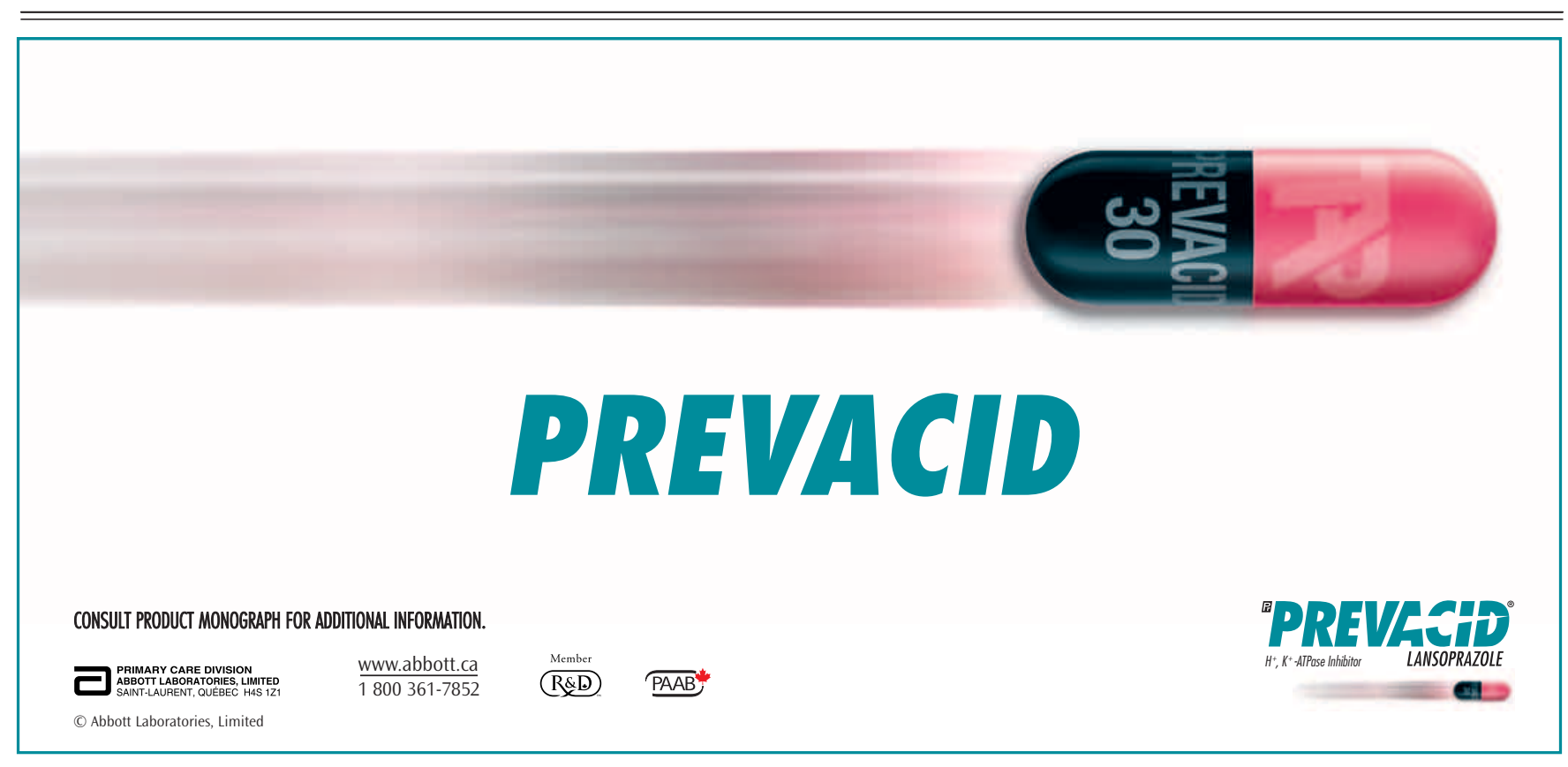






The Scientific World Journal
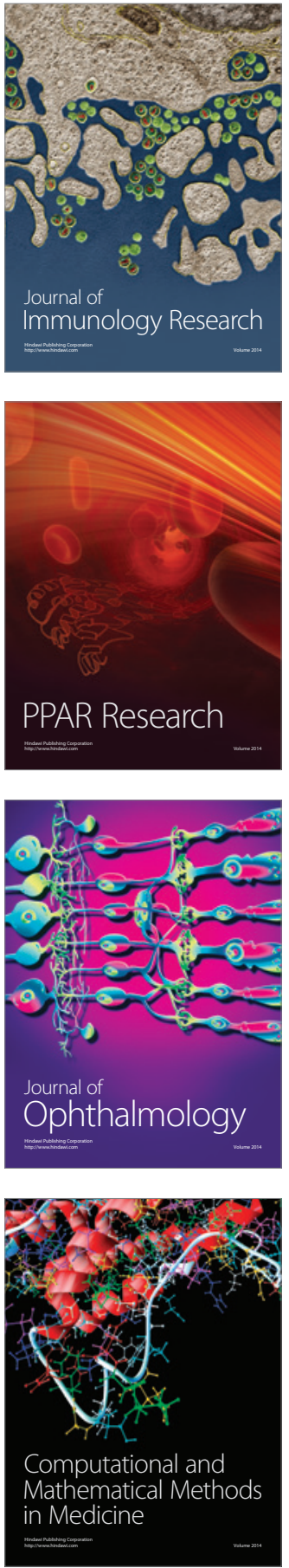

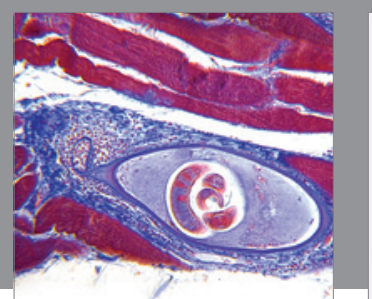

Gastroenterology Research and Practice

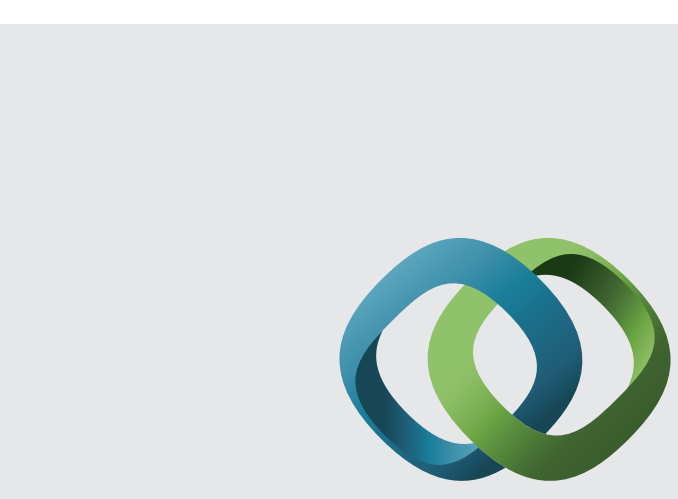

\section{Hindawi}

Submit your manuscripts at

http://www.hindawi.com
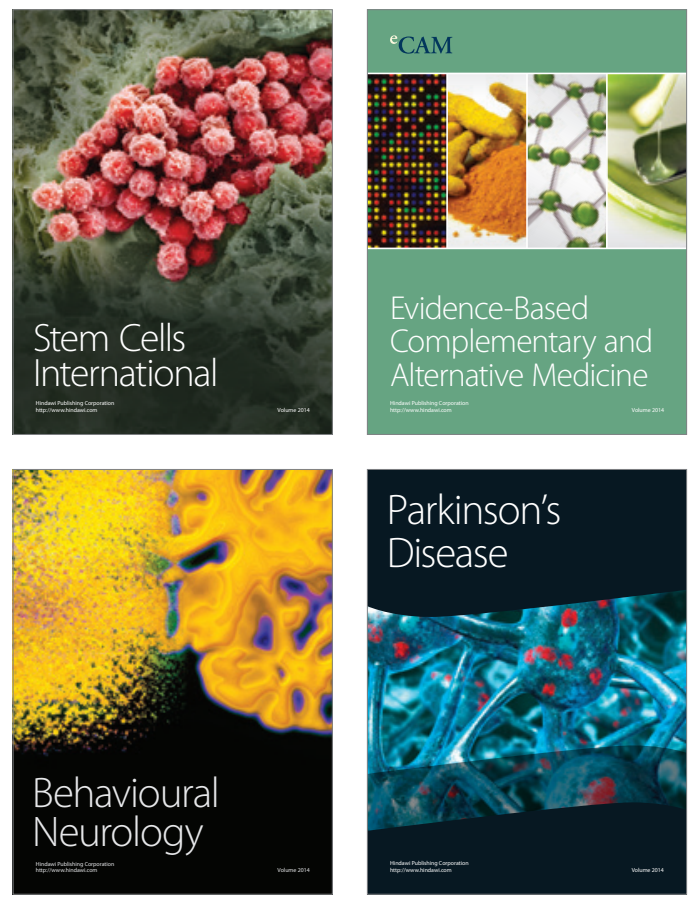


Disease Markers
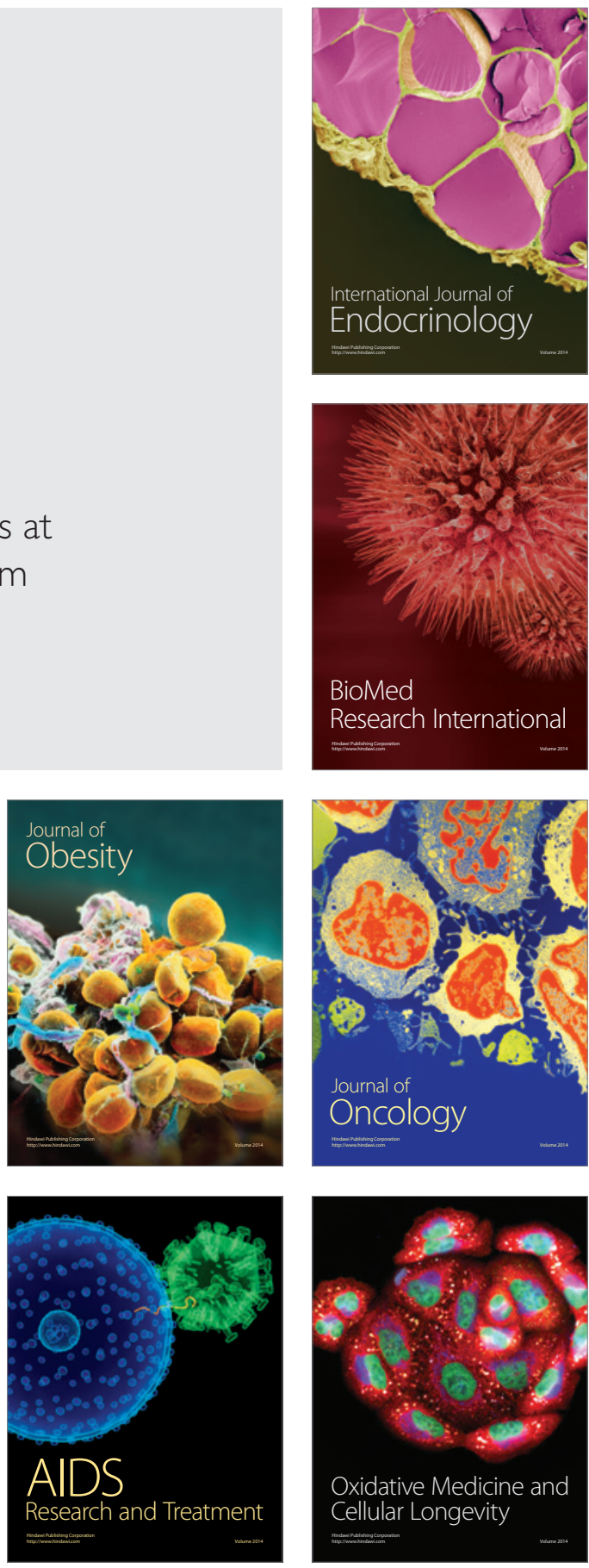